\title{
Mittel- und Neulateinische Philologie: Geschichte, Aufgabe und Erinnerung
}

\author{
Reinhard Düchting
}

Geschichte und Aufgaben der Mittellateinischen Philologie mit Erinnerungen, Geschichte und Aufgaben der Neulateinischen Philologie mit Erinnerungen - wie konnte ich nur ein solches Thema für eine halbe Stunde ausdenken und vorschlagen? Irena Radová schrieb mir: das Thema für Ihren Vortrag ist äußerst gut gewählt... Ich will Geschichte, Aufgaben und Erinnerung beider Philologien zusammen immer im Blick haben, kann aber natürlich nur wenige Punkte und gleichsam in Stichworten berühren, denke auch naturgegeben aus vorwiegend deutscher Perspektive und verbinde mit allem ganz persönliche Erinnerungen - alles Einzelne und Private muss aber für die allgemeine Geschichte und Entwicklung stehen.

Wann wird man sich seiner Geschichte bewusst?

Um 1500 sind schon Geschichten von Ordensgemeinschaften erschienen, hat der Benediktiner Johannes Trithemius literaturhistorische Kataloge (1494 und 1495) verfasst; der Humanist Konrad Celtis entdeckt in einem Kloster die Handschrift der Werke der ottonischen Hrotsvith von Gandersheim und ediert sie 1501; das staufische Epos Ligurinus eines Gunther von Pairis wird gefunden und 1507 ediert; nach Plautus im Nonnenkloster wird schon lange gesucht, die Suche nach Tacitus-Texten beschäftigt die Humanisten, die Germania wird national instrumentalisiert. Die römischen Inschriften in situ und die reichen Manuskriptsammlungen der Fugger beschäftigen die Philologen und Editoren in Augsburg vor 1600, zur selben Zeit kann in Heidelberg Janus Gruter aus dem Thesaurus der Bibliotheca Palatina edieren; Gruter ist es, der 1608ff eine erste große neulateinische Anthologie nach Nationen in 19 Bändchen veranstaltet hat: Delitiae poetarum Italorum, Gallorum, Germanorum, Belgicorum. Matthias Flacius Illyricus hatte um 1570 in 13 Bänden der sogen. Magdeburger Zenturien eine Masse älterer Schriften für den Geist der Reformation reklamiert, die katholische Kirche antwortete mit den 12 Bänden der Annales ecclesiastici des Cesare Baronius aus den Handschriften der Vaticana. Die Leistungen der jesuitischen Bollandisten (Antwerpen und Brüssel) über den Acta sanctorum und der gelehrten Mauriner um Jean Mabillon (in Paris) erhöhten im 17. Jahrhundert, auch aufgrund Routine werdender Bibliotheksreisen, das Niveau der paläographischen Forschung und Editionskultur: discrimen veri ac falsi mit dem Anspruch, die möglichst pristina forma (primigenia phrasis), die auch ein pristinus nitor ist, eines Texte zu gewinnen, wird Standard, das frühscholastische discernendum est ist eine immer gültige philologische Attitude geblieben. Im Historismus des 19. Jahrhunderts 
wird die Erforschung des Lateinischen Mittelalters durch Jacob Grimm und besonders durch das patriotische Werk der Monumenta Germaniae historica gefördert (seit 1819), mit ähnlichen Institutionen und historisch-philologischen Projekten und Interessen in Frankreich, Italien, England, auch Böhmen. Bisher sind alle Studien von Ordensleuten, von Theologen, Historikern, Juristen und (in Deutschland) Germanisten geleistet worden; wenn von Klassischen Philologen, dann bis 1900 von Graezisten.

Durch Ludwig Traube, der früh stirbt, wird Mittellateinische Philologie in München um 1900 recht eigentlich begründet hinsichtlich Paläographie, Kodikologie, Skriptorien, Abbreviaturkunde, Nomina sacra usw.; durch Traube und seine Freunde Paul von Winterfeld und Wilhelm Meyer aus Speyer wird Mittellatein auch erstmals institutionell im Kanon der Universitätsfächer verankert - in München und auch in Berlin und Göttingen. Eine Traditionsreihe entwickelte sich aber nach Traube vorerst nur in München; die zweite Institutsgründung erfolgte, promoviert von dem Latinisten Viktor Pöschl, durch meinen Lehrer Walther Bulst in Heidelberg 1955.

Bulst (†1986) war mit Ludwig Bieler und Paul Oskar Kristeller befreundet. Der gebürtige Österreicher Ludwig Bieler war 1939 nach Irland emigriert und wurde der Begründer der irischen Latinistik, des Schrifttums und aller Fragen um Pelagius und Patricius (St. Patrick), auch der Early Irish Lyrics: so kam ich auf mein Dissertationsthema über einen Sedulius Scottus, also einen Iren, der mit anderen Kollegen, die sich alle als sapientes anboten, um 850 ins Karolingerreich kam und dem Bischofshof in Leodium (Lüttich) diente als Literat, grammaticus, Exeget und Hofdichter. Bulst war in Nachfolge von Wilhelm Meyer ein großer Kenner der lateinischen Hymnodie und der historischen Entwicklung rhythmischer Dichtung, so habe auch ich mich dafür interessiert. Bulst hat sich (mit einer einzigen Ausnahme in Zusammenarbeit mit Ludwig Bertalot über Petrarca) nicht mit humanistischen oder neulateinischen Autoren und Problemen befasst - anders als ich. Kürzlich hat ein jüngerer Kollege konstatiert, ich hätte als erster in Deutschland regelmäßig Seminare über neulateinische Texte an einer Universität angeboten, vielleicht. Jedenfalls war ich mein Lebenlang mit Heidelberg in der alten Kurpfalz verbunden, der näheren Heimat des Reformators Philipp Melanchthon. Hier lebten zeitweise und starben allein zwischen 1550 und 1600 als Gräzisten, Juristen, Mediziner und Bibliothekare Jacobus Micyllus, Wilhelmus Xylander und Friedrich Sylburg, Petrus Lotichius Secundus, Johannes Posthius, Paulus Melissus Schede und Janus Gruter, in ihrem letzten Lebensjahr Olympia Fulvia Morata aus Ferrara - alle gefeierte Neulateiner, einige (Lotichius, Schede) zählen zu den besten in Europa. Ich brauchte nur in die Universitätskirche S. Peter zu gehen (und betrete sie auch noch heute fast wöchentlich), um vor den Memorialsteinen dieser gelehrten Humanisten zu stehen, und wo diese Monumenta nicht mehr vorhanden sind, sind die Epitaphien durch ein frühes Friedhofsbuch von 1612 kopial also literarisch überliefert; fast 175 Gedenksteine sind noch vorhanden. Die schriftliche Sepulchralkultur ist ein lohnendes Feld für verschiedene Studien: metrische Formen, Kunst der Abbreviaturen, Geheimnis von Chronostichen (durch rubrizierte oder erhöhte Buchstaben), lateinisch-deutsche Texte, biblische Zitate oft in anderer Schrift; man entdeckt Texte mit Akrostichen, manche haben auch noch ein Mesostichon und ein Telestichon. Die Kirchenväter (patres) um Hieronymus haben 
die Kunst der Akrostichen, die ursprünglich nicht griechisch oder lateinisch ist, in der althebräischen Dichtung der Psalmen und Lamentationes Jeremiae entdeckt, aber bei der Übersetzung der Vulgata nicht nachgeahmt (wie auch nicht die griechische Septuaginta), aber sie haben das Wissen durch Setzung der hebräischen Litterae des Alphabets (aleph, bet, gimel) vor den einzelnen lateinischen Versen markiert. Das Studium der Biblia sacra als Literatur mit den originären psalmi (sonst nur syrisch), der Formsprache der Evangelien mit Parabeln und Reden, der Apocalypse usw. für das Studium der mittellateinischen Philologie unentbehrlich ist, ebenso unentbehrlich die Patristik, in der die Weichen für Hymnodie und Hagiographie, Bibelepik, mehrfachen Schriftsinn, interpretatio Christiana usw. gelegt worden sind. Ich sprach von den Epitaphien: der polnische Jesuit Sarbiewski hat ein Carmen (II 27) geschrieben, wonach: (sibi) sepulchra et tumulos regum scholam esse - man darf hinzufügen: auch Gedenksteine von Eruditi können Lebensschule sein.

An Heidelberg grenzt die Region Kraichgau. Der Melanchthonschüler David Chytraeus hat in Rostock am Mare Balticum eine Rede auf seine Heimat De Creichgoia gehalten und 1561 in Wittenberg drucken lassen. Die Rede wurde oft zitiert nach einer ganz unzuverlässigen Ausgabe, mit einem Schüler habe ich Chytraeus faksimilieren lassen mit Übersetzung, Kommentar, Beigaben, Glossar, Literatur - damit hatte ich mir die lange und vielseitige Tradition der Descriptiones seit Flavio Biondo (um 1450) erarbeitet, über ihn habe ich das Artikelchen im Lexikon des Mittelalters geschrieben; später habe ich über die Descriptio regni Japoniae des Bernhard Varenius aus dem Jahr 1649 publiziert, Varenius ist übrigens ein Urenkel des David Chytraeus. Der vorreformatorische Frühhumanist Jacob Wimpfeling wirkte im Elsass, lange Jahre in Heidelberg und Speyer; seine Laudes ecclesiae Spirensis (auf den Speyrer Dom, 1486) habe ich mit einer Kollegin aus dem Rarissimum der Erstausgabe ediert und darin eine Menge von Plusversen gefunden, in einem Druck (der Library of Congress, Washington) haben wir ein Dedikations-Autograph von Wimpfeling entdeckt; herausgegeben habe ich auch eine seiner Heidelberger Reden De sancto spiritu (von 1486). Der Gedenkstein für Olympia Fulvia Morata und ihr schweres Schicksal hat mich schon früh und bis heute beschäftigt. Aus unserem Wohnort bei Heidelberg stammte ein berühmter Schulmann (in Zerbst, Sachsen-Anhalt) und reformierter Theologe, M. F. Wendelinus; er hat ein kleines aber dickes Buch über den Nil geschrieben; er war nie in Ägypten, hat aber aus 318 griechischen und lateinischen Büchern, die er vor sich aufbaut hatte, den gelehrten Text zu allen Fragen rund um den Nil verfasst (Admiranda Nili, 1623); auch den habe ich mit meinem Creichgoia-Schüler reproduzieren lassen und philologisch aufbereitet.

Das waren Beispiele für mein jahrzehntelanges Hineinwachsen in einige Felder des lateinischen Mittelalters und das unermessliche des Neulateinischen.

Bibliographisch gibt es reichlich Hilfe: von Medioevo Latino ist soeben (2016) der 37. Band erschienen mit 1450 Seiten Literatur mit natürlich auch historischen, theologischen, kanonistischen usw. Titeln. Auch das Neulateinische Jahrbuch bietet ein bibliographisches Instrumentum, vom Neulateinischen Jahrbuch ist soeben Band 17 (2015) erschienen. Es gibt gute Introduktionen, von J. Ijsewijn das Companion to Neo-Latin Studies (1977) und erweitert in zwei Bänden 1998-1990, soeben von M. Korenjak: Geschichte der Neulateinischen Literatur. Vom Humanismus bis zur Gegenwart (2016). Der I. Internationale Mittellateiner-Kongress 
fand 1986 in Heidelberg statt, die International Association for Neo-Latin Studies lud schon früher ein und tagt in kürzerem, dreijährigem Abstand; der letzte Kongress fand 2015 in Wien statt, der nächste schon 17. im spanischen Albacete 2018. Ich habe nur den ersten Mittellateinerkongress in Heidelberg sowie die neulateinischen in Bologna 1979, im schottischen St. Andrews 1982 und in Bonn 2003 besucht. Die Lehrmittel des Anfangs waren gering; es gab (fast) nur die Monographie des Germanisten K. O. Conrady, Lateinische Dichtungstradition und deutsche Lyrik (1961) mit einem Anhang weniger neulateinischer Texte; jahrzehntelang benutzt wurde die verdienstvolle Anthologie Lateinische Gedichte deutscher Humanisten von Harry C. Schnur aus dem Jahr 1966, soeben von einem meiner Schüler neu herausgegeben (bei Reclam 2015). Heute gibt es hervorragende, reichere und philologisch besser aufbereitete Anthologien (die ich nicht zu nennen brauche). Es gibt neulateinische Forschungszentren, immer noch Löwen (Louvain), Florenz, seit einigen Jahren Innsbruck, auch in Heidelberg um Wilhelm Kühlmann und seinen Schülerkreis. In Florenz (durch die Stiftung SISMEL: der Societá internazionale per lo studio del medioevo latino) und in Turnhout (Belgien) stehen potentielle Offizine für kleinere und größere Editions-Serien zur Verfügung; de facto muss kein gelehrtes Werk ungedruckt bleiben, die Gelder können meistens beschafft werden.

Ich will mit einem Monitum und zuversichtlich schließen.

Die Klage: seit den Anfängen der beiden Philologien um 1900 und nach 1950 ist es nicht gelungen, das Fach Mittellatein und Neulatein als eigenständiges Universitätsfach zu etablieren. In München ist Mittellateinische Philologie nur noch durch eine wesentlich philosophiegeschichtlich ausgerichtete Professur innerhalb der Klassischen Philologie vertreten, in Heidelberg durch einen Privatdozenten bei den Historikern; soeben höre ich, dass das Marburger Seminar ganz geschlossen wurde, woanders ist Mittellatein Teil eines interdisziplinär arbeitenden mediävistischen Verbands oder der erweiterten Altertumswissenschaften. Die Gründe dieser Lage sind oft beschrieben worden: wer Mittel- (und Neu)Latein studiert, hat in der Regel zuvor andere Fächer studiert (ich Evangelische Theologie und Germanistik), deren akademischer Abschluss die Übernahme in den Staatsdienst garantiert. Mittellatein dagegen können auch Klassische Philologen, Historiker, Romanisten, Archivare und Bibliothekare studieren und könnten es noch besser, wenn sie alle Leistungen und Kenntnisse der Mittellateinischen Philologie immer zur Kenntnis nehmen würden. Trotz aller angebotenen, ausdifferenzierten Ausbildungsmöglichkeit mit allen möglichen Modulen bleibt die Frage, wohin die erworbene Kapazität die das Fach Studierenden führt; die Schule hatte sich nie (außer in wenigen historischen Momenten) und wird sich heute und in Zukunft angesichts der Lernreduzierung und schwindendem Interesse dem Fach Mittel- und Neulatein nicht öffnen, auch wenn der Fachlehrer lateinische Texte des Mittelalters und der Frühen Neuzeit mit Erfolg anbieten kann. Die Universität selbst kann in ihrem Stellenpool kaum attraktive Angebote machen; diese Sorge aller interessierten und begabten Studierenden ist auch die Sorge aller Verantwortlichen.

Gleichwohl will ich uns alle, Lernende und Lehrende, ermuntern mit einem Dictum des schon genannten Augsburger Gräzisten Xylander (Holtzmann) in Heidelberg. Als der Kurfürst Friedrich III. (der des Heidelberger Katechismus) 1569 seine Professoren auf- 
fordert, über akademische Disziplin, Regelmäßigkeit der Vorlesungen, Frequenz der Studenten usw., Rechenschaft zu geben, antwortete er schriftlich (Jana Nechutová kennt den Text aus Sibi et amicis):

„die Anzahl der Zuhörer habe ich niemals nachgezählt und glaube auch durchaus nicht, dass dies der Würde eines öffentlichen Professor zukommt, der sich einer großen Zahl nicht brüsten darf (solches erregt nämlich den Neid der Collegen, ist zudem schwankend) und den auch nicht eine kleine Zuhörerschaft auszeichnet" wo es doch vor allem einem guten Magister zukommt, seinen Lehrvortrag nicht einer Zahl sondern dem Gegenstand anzupassen und nicht etwa nach Schülern ausschauzuhalten oder um sie zu buhlen, vielmehr die, die kommen, recht und mit gutem Gewissen zu lehren. Im Übrigen, wie die Verhältnisse und Zeiten sind, brauche ich mich meines Auditoriums nicht zu schämen

praesertim cum boni magistri sit, vocem in docendo non numero sed rei accomodare et non quaerere aut ambire discipulos, sed qui auditum veniunt, eos rectè ac bona cum conscientia docere. Interim, ut res sunt et tempora, non habeo, ut me mei poeniteat auditorii.

Prof. Dr. Reinhard Düchting / dduechting@freenet.de

Lateinische Philologie des Mittelalters und der Neuzeit am Historischen Seminar

Ruprecht-Karls-Universität Heidelberg, Philosophische Fakultät

Grabengasse 3-5, 69117 Heidelberg, Deutschland 
